\title{
CALC, UN OUTIL DE TRAÇABILITE EN CONCEPTION
}

\author{
E. Ostrosi", O. Garro", A. Koukam" et D. Brissaud"
}

Résumé. - Une entreprise qui souhaite améliorer ses futures conceptions en réutilisant des connaissances acquises dans les conceptions présentes se doit de conserver le produit lui-même, ce qui se fait normalement, mais aussi la logique de conception ayant amené à ce produit, ce qui ne se fait jamais. Cet article présente un cadre formel et un outil pour la prise en compte d'informations portant sur la logique du processus de conception. Ces informations doivent être structurées et organisées pour être utilisées dans des projets de conception futurs et éventuellement pour justifier les décisions prises lors des évolutions futures du projet. Nous examinons tout d'abord le mouvement conjecture-critère sur lequel est basée cette logique de conception et nous montrons qu'il est possible de conserver des informations permettant de tracer cette logique de manière relativement exhaustive. Nous présentons ensuite différents niveaux de représentation de ces informations suivant la réutilisation envisagée. Cette structure est implémentée dans le prototype informatique CALC (Outil d'Aide à la CApitalisation de la Logique de Conception), dont l'usage est discuté.

Mots-clés :

\section{Introduction}

Depuis plusieurs années, il est reconnu que la conception est distribuée, parallèle et intégrée. La distribution porte sur les métiers, les ressources, les connaissances et les acteurs. Le modèle de conception distribuée, mis au point dans [Garro 95] montre d'une part la répartition des métiers en modules de connaissances et d'autre part la structure des langages manipulés par

\footnotetext{
'Université de Technologie de Belfort - Montbéliard Mecatronique 3M F 90010 - Belfort Cedex Tél: 0384 583136 / Fax: 0384583146 E-mail : Egon.Ostrosi@utbm.fr.

"Institut Polytechnique de Grenoble 46, avenue Félix Viallet 38031 Grenoble Cedex 1 Tél. 0476574500 Fax. 0476574592
} 
ces métiers. Chaque métier a ses propres objectifs. Il va donc mobiliser des logiques différentes pour aboutir au choix des solutions. Intégrer un métier, c'est lui permettre de défendre son point de vue sur le futur produit ; c'est donc le faire participer aux prises de décision et au choix des solutions qui l'engagent [Laureillard 97],

De nombreux travaux portent sur la mise en commun, au sein de bases de données techniques, du produit à concevoir en prenant en compte les différents points de vue des acteurs concernés par l'ensemble du cycle de vie du produit. Ceci passe par la définition de modèlesproduits multi-vues, multi-utilisateurs et multi-sites [Tichkiewitch 91] d'une part, ou de systèmes automatiques de reconnaissance d'entités spécifiques à chaque métier d'autre part [Ostrosi 98]. Dans notre optique, la direction de recherche la plus prometteuse porte sur les entités (features) qui permettent d'associer à la forme le savoir-faire du concepteur et qui sont une représentation adaptée pour que les spécialistes des métiers de la conception puissent construire leur représentation dans leur propre logique. Il s'agit d'une première approche pour l'intégration des points de vue en conception.

Ce premier aspect n'est cependant pas suffisant [Duffy 99]. El permet de modéliser le produit en cours de conception de manière satisfaisante et utile pour tous les acteurs du cycle de vie du produit ; c'est une avancée importante. En revanche, il ne permet pas de tracer les stratégies mises en œuvre par l'ensemble des acteurs pour choisir les solutions. Or nous avons besoin de comprendre ces stratégies pour comprendre la solution finalement retenue. Lors d'un projet, le besoin d'information porte donc à la fois sur l'évolution de la solution courante et sur les logiques et les raisonnements mis en œuvre par les concepteurs pour faire évoluer cette solution [Karsenty 94]. C'est sur ce point particulier que nous insistons dans ce texte. Que doiton garder pour reconstruire la logique de conception? Qu'est-on capable de garder ? Comment pourra-t-on les réutiliser en pratiques industrielles de conception?

Les travaux que nous présentons sont le résultat d'analyses d'expériences de conception [Blanco 98]. Dans ces expériences, un certain nombre d'acteurs (de 3 à 5) jouent le rôle de techniciens en charge de métiers particuliers (fabrication, calcul...) et conçoivent ensemble un produit novateur défini par un cahier des charges initial.

Dans la deuxième partie, nous présentons les données propres à la logique de la conception. Ici, nous introduirons la notion de mouvement conjecture-critère qui constitue la base de proposition d'une structure des données de conception.

La troisième partie présente les différents niveaux de représentation de la logique de conception, qui définit un cadre structuré pour la prise en compte d'informations portant sur la logique du processus de conception.

La quatrième partie présente le logiciel prototype CALC (Outil d'Aide à la CApitalisation de la Logique de Conception) qui permet le suivi des informations relatives à la démarche de conception d'un produit, selon les différents niveaux de représentation de la logique de conception présentée à la deuxième partie. 
Ensuite, dans la cinquième partie, nous discutons sur le cadre présenté et sur les outils possibles d'aide à la rationalisation de la conception. En conclusion, nous insisterons sur la nécessité d'instrumentalisation du processus de conception.

\section{Les données propres à la logique de conception}

\subsection{Analyse d'un processus de conception}

Parmi les nombreuses tâches que réalisent les concepteurs (planification, représentation...), il en est deux qui sont d'une grande importance pour l'avancement de la conception, notamment pour l'innovation et l'apparition de nouvelles solutions.

La première tâche est la proposition de nouvelles solutions par un concepteur. La seconde est l'évaluation de ces solutions. Ceci se fait au travers d'allers retours entre le monde des problèmes et le monde des solutions. Cette boucle élémentaire est présentée sur la figure 1.

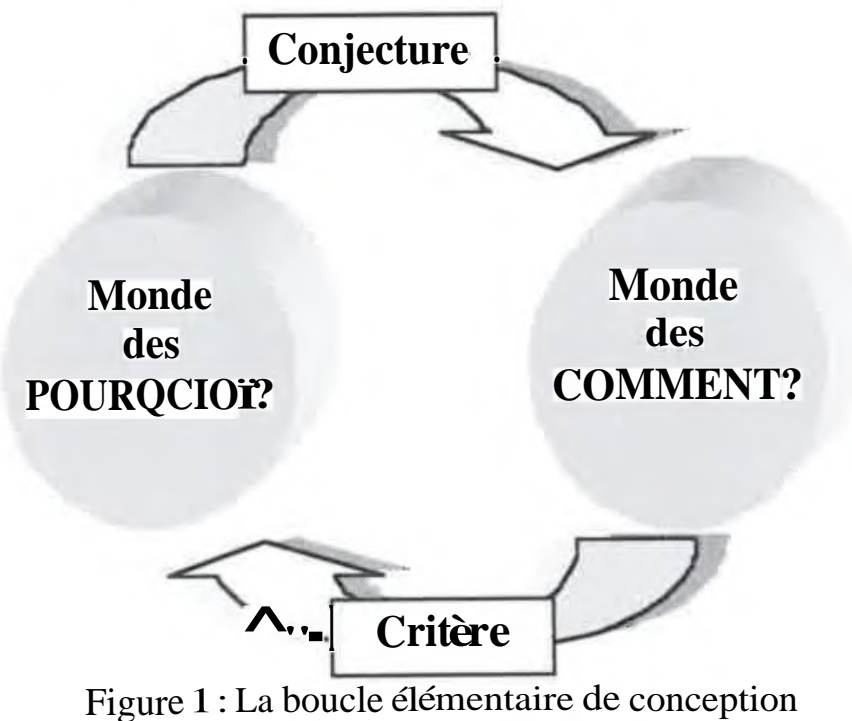

Le Monde des Pourquoi constitue l'espace des problèmes. Il est généralement décrit sous forme fonctionnelle, par un cahier des charges fonctionnel. On demande aux concepteurs de ne pas faire mention de moyens ou de solutions lors de l'écriture de ce cahier des charges. Il est aujourd'hui reconnu qu'au cours de la conception, la description du problème va évoluer en se précisant et aussi en se modifiant; le cahier des charges fonctionnel va donc suivre cette évolution.

Le Monde des Comment constitue l'espace des solutions. Ce aussi bien sont des solutions physiques (comment dois-je réaliser ma fonction ?) que des solutions en termes de processus (comment dois-je réaliser ma forme ?). Dans ce monde des solutions, on va également trouver des solutions déjà éprouvées sur d'autres problèmes. Ces solutions plus ou moins bien définies peuvent être utilisées par les concepteurs pour progresser. Dans ce monde, au cours du processus de conception, la définition des solutions va se préciser et s'affiner. 
Ainsi, au cours du processus de conception, le problème et la solution vont évoluer conjointement. Cette dynamique se produit grâce au double mouvement conjecture / critère.

Le premier mouvement est la proposition d'une conjecture. Une conjecture est une entité qui appartient au monde des comment. Il s'agit d'une solution potentielle, candidate à devenir solution entière (ou partie de solution). Une conjecture est proposée par un concepteur à un moment donné de la conception. Elle répond partiellement au problème, puisqu'elle est émise sous les contraintes du problème tel que les perçoit le concepteur à ce moment-là. Une conjecture, si elle est acceptée par les autres acteurs, va devenir une solution courante du problème.

L'évaluation constitue le second mouvement. Pour être acceptée, une conjecture est évaluée. L'évaluation se fait par des critères. C'est une entité qui relève du monde des pourquoi. Le critère est proposé par un concepteur en réaction à une conjecture. Un critère peut être de toute nature. Il est abstrait ou opérationnel, précis ou flou, quantitatif ou qualitatif... Ce qui est important, c'est qu'il va permettre aux concepteurs de faire des choix parmi les différentes conjectures. En général les concepteurs mobilisent plusieurs critères pour évaluer une conjecture.

Les critères sont des justificatifs à un avis. Ils sont appliqués avec un sens. Ce sens est soit positif, soit négatif ; il conforte la conjecture proposée ou la remet en question. Les critères sont rarement quantifiés; il s'agit généralement d'appréciations qualitatives et subjectives.

Remarque: Un critère est de même nature qu'une contrainte. Si cette entité est utilisée pour délimiter le problème, il s'agit d'une contrainte. Si elle est utilisée pour évaluer une conjecture ou une solution, c'est un critère. Nous reviendrons plus en détail sur les critères qui se trouvent au cour des propositions que nous formulons. Ce qui est important ici, c'est que c'est dans ce mouvement conjecture critère que la conception se fait.

L'interaction de base entre conjecture et critère est donnée par la figure 2. Un acteur $\boldsymbol{i}$ propose, à une date $p$, une conjecture relativement à une entité en débat. Un acteur $j$ réagit à cette proposition et avance un critère d'évaluation de la conjecture proposée, à la date $q$. Ce critère donne un avis positif ou négatif sur la conjecture (le sens du critère).

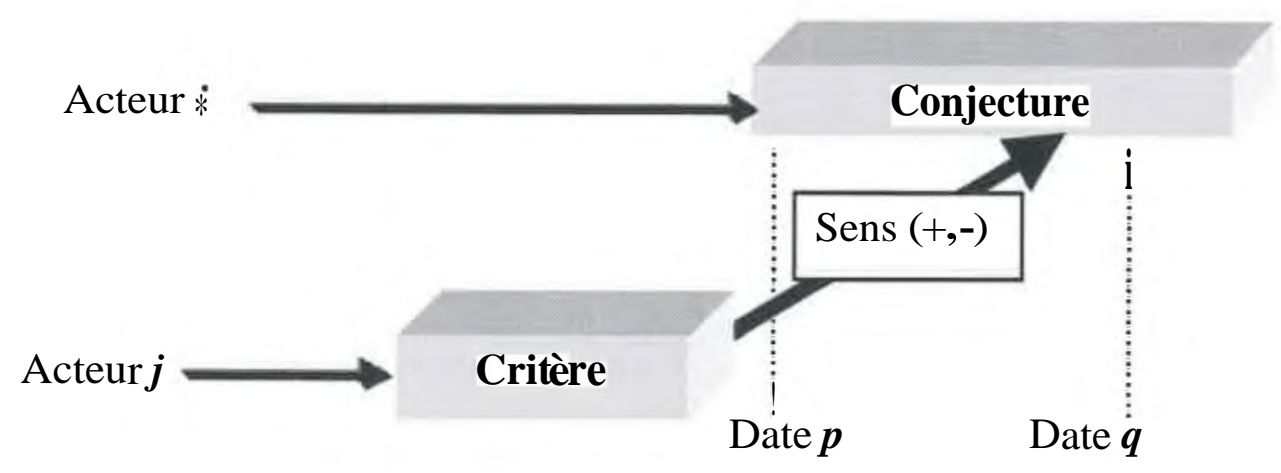

Figure $2:$ Interaction conjecture - critère. 


\subsection{Structure des données du processus de conception}

Les données mises en œuvre dans l'interaction conjecture - critère sont triples. Il y a tout d'abord les données propres à l'interaction. Ces données ne sont accessibles que pendant l'interaction entre les concepteurs; il s'agit donc de les capter en dynamique. Nous avons ensuite les données qui sont connues et qui doivent être obtenues préalablement à l'interaction. Enfin, d'autres données résultent de la dynamique d'interaction et peuvent être construites $a$ posteriori.

\subsubsection{Les données accessibles dans l'interaction}

Les données mises en œuvre lors de cette interaction sont:

- la conjecture : c'est une entité sur laquelle un concepteur cherche à faire prendre une décision;

- l'acteur qui émet la conjecture;

- la date à laquelle la conjecture a été émise;

- le critère;

- l'acteur qui émet ce critère;

- la date d'émission de ce critère;

- le sens du critère.

Toutes ces données sont disponibles pendant les échanges entre concepteurs. Ces données se rapprochent du modèle QOC (Question, Option et Critère) développé par [Mc Lean 96]. Le modèle QOC permet de produire une représentation graphique de la logique de conception. Dans ce modèle semi-formel, une Question permet d'identifier les problèmes clés de la conception. Une Option fournit des alternatives relatives aux Questions, donc aux problèmes identifiés. Un Critère permet l'évaluation et la comparaison de chaque Option envisagée. Le formalisme QOC est destiné à faciliter la réutilisation d'une solution mais aussi la réutilisation d'un raisonnement. Mais ce modèle se veut général parce qu'il n'a pas pour vocation de retenir toutes les spécificités de chaque domaine de conception [Karsenty 00]. Notre modèle s'éloigne du modèle QOC sur deux points. D'une part, les questions sont rarement formulées de manière explicite par les concepteurs et, d'autre part, le traitement, que nous présentons par la suite, au niveau des données, va être très différent.

\subsubsection{Les données disponibles avant l'interaction}

Les données disponibles au préalable sont les données relatives au projet; l'organisation de la réunion et les participants. Un participant représente un métier particulier. Ce métier a des responsabilités spécifiques dans la conception, ce qui l'autorise à être expert dans certains domaines de connaissance appelés registres de référence. Un registre de référence est un domaine de connaissance dans lequel les critères vont trouver leur justification. 


\subsubsection{Les données construites après l'interaction}

Ces données sont construites à partir de données observées pendant l'interaction. Il s'agit essentiellement de l'appartenance d'un critère à un registre de référence.

La liste des critères ne peut pas être finie, puisque ceux-ci sont construits dans l'interaction; ils ne peuvent donc pas être prédéfinis. En contrepartie, la liste des registres de référence est finie [Blanco 98]. On trouvera parmi les registres de référence la fabrication, l'économie, le domaine juridique, etc.

Cependant, associer un critère à un registre de référence tient de l'interprétation. Alors que toutes les données étaient jusqu'à présent objectives, on commence à rentrer dans une classification subjective. L'intérêt de ce lien est cependant important. La comparaison du registre de référence du critère avec le métier de l'acteur va permettre de tester le degré d'expertise de l'acteur. Ceci va participer au poids donné à ce critère par l'ensemble d'acteurs.

A partir de ces trois types de données, nous pouvons créer une base de données connectée à la base de données du produit (Modèle Produit par exemple) par les conjectures. La structure de ces données est présentée par la figure 3.

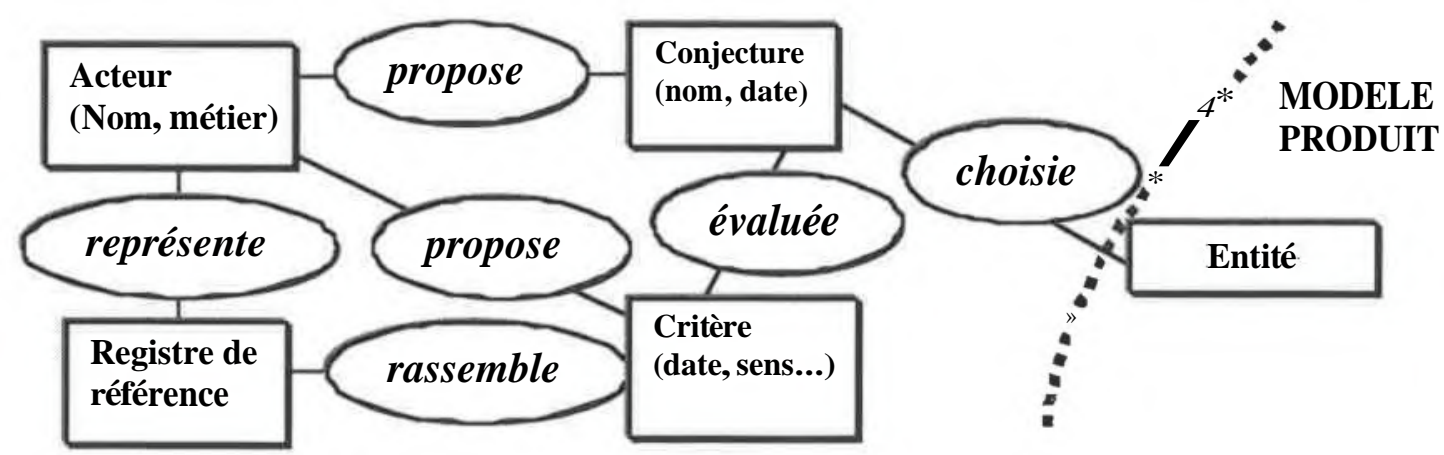

Figure 3 : La structure des données brutes d'analyse de la conception

A partir de ces données, on peut donc reconstruire la prise de décision qui a conduit au choix d'une solution.

\section{Différents niveaux de représentation de la logique de conception}

Dans un projet, qui comprend plusieurs réunions et une grande quantité de travail hors réunion, le nombre d'interactions de type conjecture/critère est considérable. Il n'est pas nécessaire de fournir aux concepteurs l'ensemble de ces données brutes, soit parce qu'elles sont peu pertinentes pour l'exploitation attendue dans le processus de conception de produits futurs, soit parce qu'elles sont trop riches pour pouvoir être exploitées et stockées. Il est donc important de pouvoir accéder à des niveaux de représentation plus synthétiques. Nous proposons cinq niveaux de représentation (figure 4). Ces niveaux vont du plus riche au plus synthétique. 


\section{Niveau 5}

Niveau 4

Niveau 3

Niveau 2

Niveau 1

\section{Projet}

\section{Critères}

\section{Solutions}

\section{Conjectures}

\section{Données Brutes}

Figure 4 : Différents niveaux de représentation de la logique de conception.

\subsection{Niveau des Données Brutes}

Ce niveau est constitué de toutes les informations décrites précédemment. Il représente le modèle complet des informations permettant de justifier la logique de conception.

Par exemple, dans l'interaction suivante entre trois concepteurs :

- Acteur 1 : la solution serait de prendre une forme ronde

- Acteur 2 : carrée à coins arrondis ?

- Acteur 1 : c'est plus difficile à usiner.

- Acteur 3: mais c'est moins agressif pour l'opérateur.

Les données brutes peuvent être représentées par la succession :

- (Acteur 1 , date i : conjecture = forme ronde $)$

- (Acteur 2, date $\mathrm{i}+1$ : conjecture = forme carrée à coins arrondis)

- (Acteur 1 , date $\mathbf{i}+2$ : critère = usinage, sens négatif sur conjecture $=$ forme carrée à coins arrondis)

- $\quad$ Acteur 3 , date $i+3:$ critère $=$ forme non agressive, sens positif sur conjecture $=$ forme carrée à coins arrondis). 


\subsection{Niveau des Conjectures (figure 5)}

Pour le second niveau, nous proposons de supprimer les données qui ne nous semblent pas directement pertinentes pour notre problème. Il s'agit de l'acteur (cette information est difficilement exploitable dans les projets futurs et peut être source de problèmes sociaux par la suite) et des dates (sans réel intérêt ici). Si nous proposons d'oublier l'acteur qui propose la conjecture, nous suggérons de remplacer l'acteur qui émet un critère par une notion d'expertise. Cette notion d'expertise portée par l'acteur intervient dans une notion plus globale de crédibilité du critère. Cette expertise résulte de l'analyse de la boucle acteur - métier - registre de référence critère - acteur (figure 3 ).

A ce niveau, on obtient donc, pour chaque conjecture, l'ensemble des critères évoqués pour l'évaluer. Chaque critère est qualifié par son sens et par sa crédibilité. Parmi l'ensemble des conjectures proposées, une d'entre elles va être choisie comme solution et représentera une entité du produit.

Ce modèle peut donc être utilisé pour retrouver les solutions intermédiaires étudiées et les raisons du choix de la solution retenue.

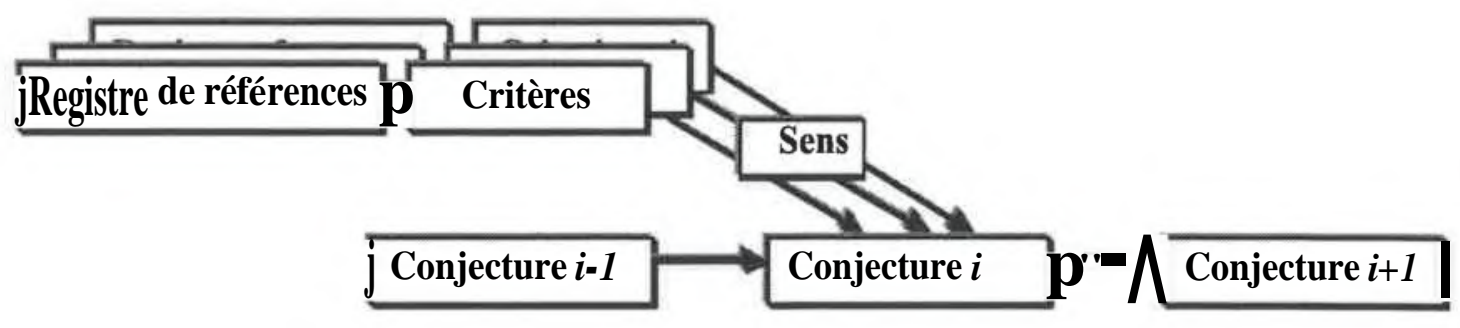

Figure 5 : Evolution des conjectures

Si nous reprenons notre exemple, cela nous donnera :

(Conjecture $\mathbf{1}$ = forme ronde, pas de critères)

(Conjecture 2 = forme carrée à coins arrondis, critère 1 : usinage, sens négatif, registre de référence : fabrication, émis par expert, critère 1 : forme non agressive, sens positif, registre de référence : ergonomie, émis par non-expert).

\subsection{Niveau des Solutions}

Dans ce niveau, nous supprimons toutes les conjectures. Il ne reste alors que la solution retenue associée aux critères évoqués avec leur sens et leur notion de crédibilité. 
Chaque solution-entité est isolée avec les critères qui lui ont été appliqués (figure 6). Ceci permet, dans le cas où l'on veut modifier une entité particulière, d'analyser l'ensemble des critères qu'elle met en jeu et d'évaluer les modifications de performances qui découleront de la modification de cette entité. Ce point de vue peut être intégré directement dans le modèle produit puisqu'il est attaché à une entité de ce modèle produit.

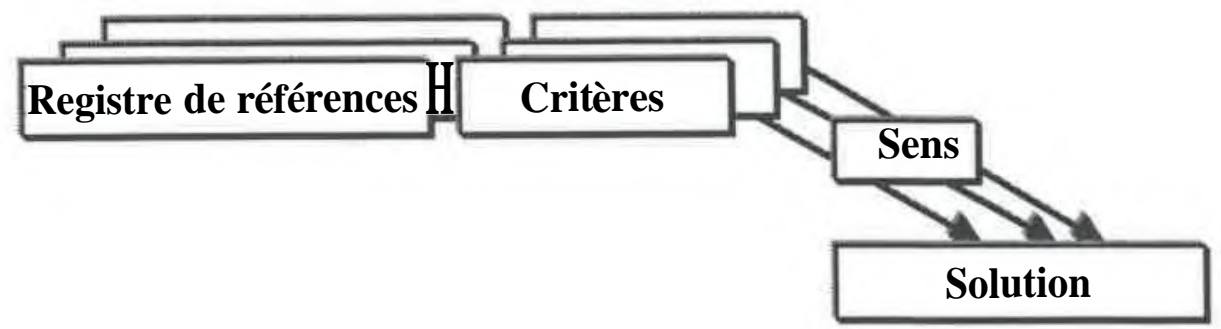

Figure 6 : Niveau des solutions

Il est intéressant de récupérer les critères portés sur les autres conjectures afin de les appliquer à la solution finale. En général, un critère négatif porté sur une conjecture intermédiaire sera positif pour la solution choisie. Inversement, un critère positif intermédiaire sera souvent négatif pour la solution retenue. Ced n'est cependant pas systématique. Il convient ici de procéder à un traitement intelligent de cette agrégation de critères. Ceci introduit une interprétation supplémentaire. Il serait intéressant de rajouter un marqueur sur le sens du critère. Ce marqueur doit relativiser le classement des critères et permettre au concepteur de remonter au niveau 2 pour analyser plus finement le sens des critères si nécessaire.

Dans l'exemple que nous venons de prendre, aucune des conjectures citées n'a été retenue comme solution. La solution est :

Solution : carrée.

Par contre les critères émis sur les conjectures peuvent être réutilisés en les inversant :

Autre critères : critère 1 : usinage, sens positif, registre de référence : fabrication, émis par expert, critère 1 : forme non agressive, sens négatif, registre de référence : ergonomie, émis par non-expert.

Dans l'outil que nous avons développé, à partir de ce niveau, nous générons directement le compte rendu de réunion. Dans ce compte rendu, les solutions retenues et les raisons de ces choix sont présentées.

\subsection{Niveau des Critères}

Chaque critère est isolé avec les solutions-entités qui en dépendent; le sens et la crédibilité du critère restent associés au lien qui les unit (figure 7). Ceci permet d'analyser les solutions- 
entités qui améliorent ce critère et celles qui le détériorent. On peut ainsi améliorer le travail de reconception lorsque l'on a un objectif de performance bien précis (par exemple, diminuer le poids).

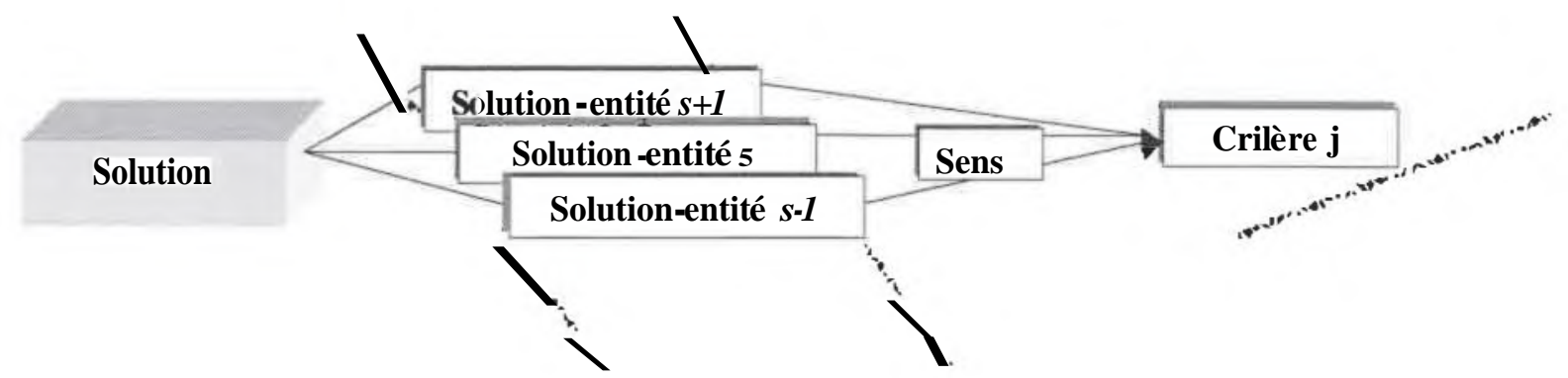

Figure 7 : Niveau des critères.

\subsection{Niveau du Projet}

Ce niveau est le plus synthétique. Il reste d'une part la solution et d'autre part les critères. L'entrée par la solution rejoint le cas classique dans lequel on visualise la solution retenue. C'est la raison pour laquelle nous n'en discuterons pas davantage. L'entrée par les critères est plus originale. Il s'agit de la liste de tous les critères mis en ouvre dans une conception. Cette liste a trois intérêts. Elle peut servir de point de départ à une analyse du travail de conception : est-ce que certains critères ou certains registres de références ont été oubliés ou au contraire trop souvent mis en avant? Mais elle peut aussi être capitalisée en vue de servir dans d'autres conceptions ou reconceptions. Enfin, elle peut servir à des fins d'argumentaire commercial ou de marketing.

\section{CALC: Outil d'Aide à la CApitalisation de la Logique de Conception}

CALC est un outil d'aide à la capitalisation de la logique de conception distribuée. H permet le suivi des informations relatives à la démarche de conception d'un produit. Un projet de conception doit permettre l'élaboration d'un produit; pour cela, des acteurs se rassemblent au cours de réunions. Lors de ces réunions, des idées sont émises; ce sont ces informations qui nous intéressent pour ainsi suivre la démarche de conception de l'idée première jusqu'au choix d'une solution. CALC permet de saisir toutes ces informations et de les organiser en vue de leur consultation [Mettavant 99]. Le modèle conceptuel de CALC est représenté par le diagramme de flot de données (figure 8).

(C) Revue Française de Gestion Industrielle - Vol 20, Nº1 


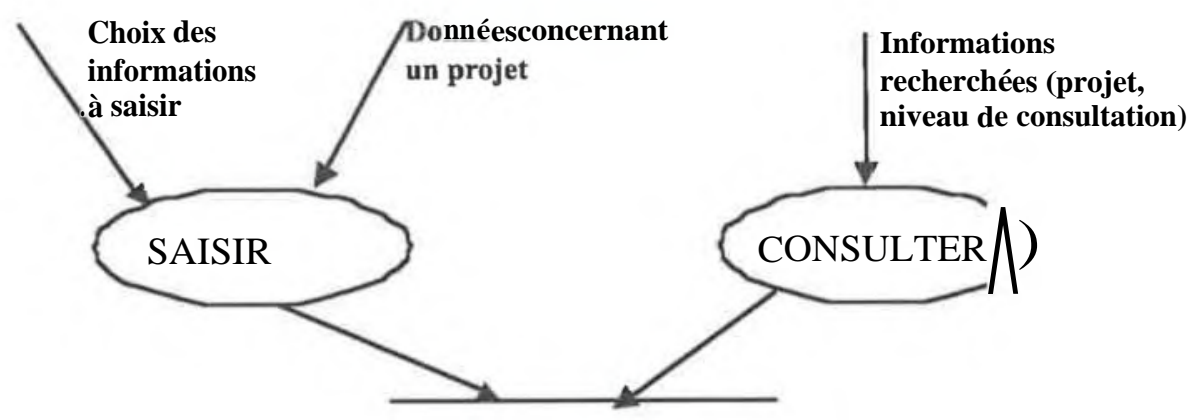

BASE DE DONNEES

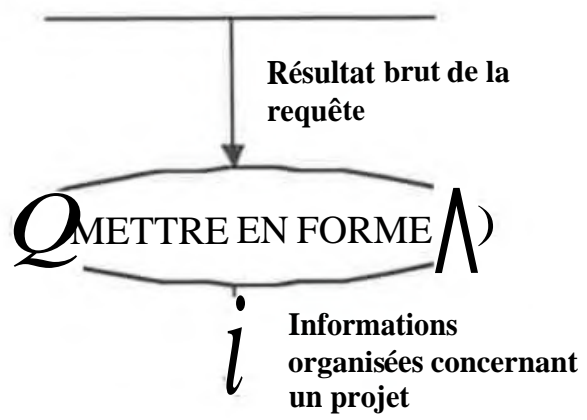

Figure 8: Modèle conceptuel de CALC

La base de données est installée sur une machine comportant Oracle Web Serveur. L'application fonctionne en client-serveur. Sa réalisation en HTML a permis Tutilisation d'un navigateur WEB (browser) et donc l'affichage des écrans d'interfaces homme-logiciel (figure 9). Des procédures stockées dans la base de données permettent la génération dynamique des pages HTML, en fonction des appels. Ces procédures sont implémentées en PL/SQL, langage qui a permis l'insertion des requêtes SQL sur la base de données.

L'utilisateur de CALC a deux possibilités en entrée. Soit il fait de la saisie de données brutes de conception (par exemple en réunion), soit il consulte les données d'une réunion ou d'un projet.

La saisie en réunion vise à entrer les données brutes. Les différents écrans de saisie offrent plusieurs choix à l'utilisateur : quel est l'acteur qui émet, qu'est-ce qu'il émet (critère ou conjecture) ? Dans le cas d'un critère, quel est son sens et sur quoi porte-t-il ?

Un deuxième mode de saisie permet d'entrer avant un projet ou une réunion la liste des acteurs et leur appartenance ou leur expertise dans un registre de référence.

Enfin, un troisième mode de saisie permet de relier les critères qui ont été émis a des registres de référence. 
La consultation consiste à naviguer dans les différents niveaux afin de visualiser les enchaînements de critères et de conjectures. Les cinq niveaux sont accessibles soit pour une réunion précise, soit pour l'ensemble d'un projet.

La distribution de ces informations sur l'intranet a pour objectif de donner à tous les acteurs d'un projet, d'une part, des informations sur les décisions qui ont été prises en cours de projet et, d'autre part, des informations sur les alternatives (conjectures) proposées par rapport à une solution qui a été choisie.

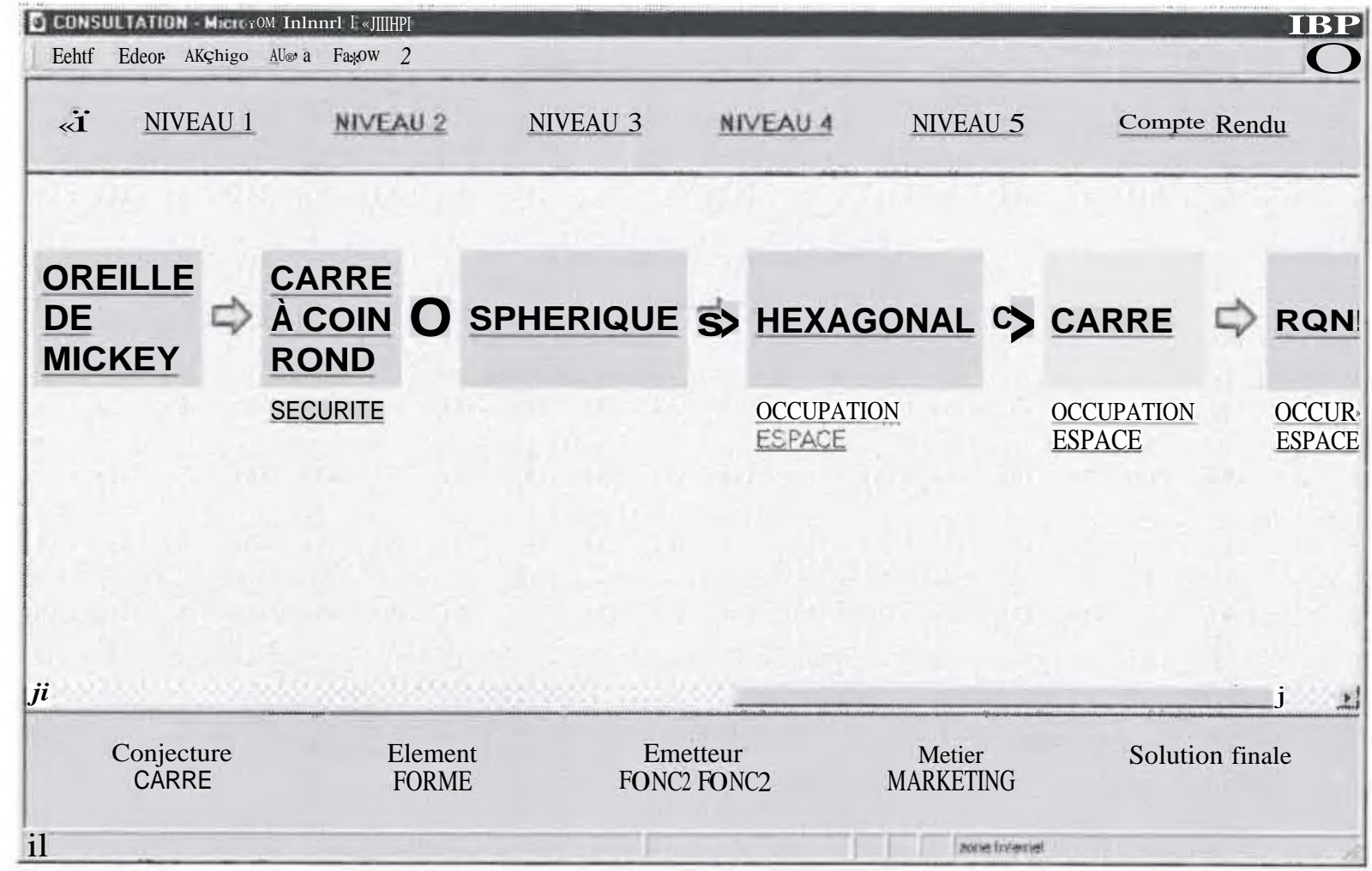

Figure 9 : Interface homme-logiciel

\section{Discussion sur les niveaux et les outils possibles}

Nous avons montré dans ce texte la structure de l'émergence des solutions. Nous avons également montré l'importance du rôle des critères. Ceux-ci peuvent servir à comprendre la genèse des solutions. Les différents niveaux de représentation peuvent donc servir à :

- analyser le processus de conception,

- évaluer le choix d'une solution,

- aider à la reconception d'une partie ou de toute une solution selon un objectif de performance précis (entrée par un critère ou un groupe de critères) ou selon un besoin technique (modification d'une forme particulière), 
- capitaliser le savoir-faire des concepteurs.

Les limites de cet outil sont cependant importantes. Il est nécessaire d'en tenir compte.

- Dans un premier temps, nous avons mis en évidence les différentes interprétations qu'il est nécessaire d'introduire pour atteindre chacun des niveaux du modèle. Plus le niveau est élevé et plus les interprétations sont importantes.

- Ensuite, il faut avoir conscience que tous les critères partagés par les concepteurs n’apparaissent pas dans les interactions. Une partie de ces critères peut être retrouvée par des techniques d'entretien. Cependant, on peut penser qu'il y aura toujours des critères qui échapperont à toute enquête, soit parce qu'ils sont ignorés par les concepteurs, soit parce qu'ils ne sont pas explicitables, soit encore parce qu'ils sont également partagés par l'enquêteur.

- La difficulté la plus grande demeure cependant la récupération des critères. Les travaux que nous avons menés ont toujours été conduits sur des expériences filmées avec retranscription des bandes audio. Outre la lourdeur du dispositif, il semble difficile de le mener à bien systématiquement. Une autre solution consisterait à introduire en permanence un nouvel acteur-observateur. Ce nouvel acteur de la conception aurait pour mission de prendre en note l'ensemble des débats entre concepteurs. Cette approche mérite d'être testée.

La seule solution viable à notre sens et qui pourrait intégrer les deux dernières objections serait d'instrumenter le processus de conception afin de récupérer le plus possible de critères. Deux situations sont possibles, à savoir :

i. Comment collecter les critères mobilisés par un acteur travaillant seul sur une partie d'un projet. Jusqu'à présent, nous n'avons évoqué que le travail en groupe. Or, en conception distribuée, une grande partie du travail se fait séparément, chacun mobilisant dans son coin les outils de son métier particulier afin de produire un résultat qui servira à l'équipe projet. Nous pensons que c'est à ce concepteur particulier que doit revenir la tâche de donner les critères de ses choix en même temps qu'il apporte ses décisions. Tout résultat doit donc être accompagné d'un nouvel objet qui prendra en compte plusieurs des niveaux décrits précédemment. Il y aura les différentes conjectures analysées et les critères de rejet de ces conjectures, ainsi que les critères positifs. Un tel objet peut donner lieu à un cycle auteur-lecteur. Ceci peut avoir plusieurs avantages. Tout d'abord, cela peut obliger chaque concepteur à explorer plus en profondeur ses choix et à se forcer à établir plusieurs solutions. Ensuite, si le lecteur est d'un autre métier, l'auteur sera obligé de mieux expliciter son travail. Enfin, et c'est pour nous le plus important, un tel objet serait source d'interactions multiples en obligeant les concepteurs à sortir de leurs métiers afin de croiser leurs points de vue.

ii. Comment collecter les critères mobilisés par un groupe. Ici, les outils à mettre en œuvre sont plus complexes. Plusieurs solutions sont possibles. Nous névoquerons pas des solutions technologiquement lourdes comme la reconnaissance vocale et l'analyse automatique de corpus. A notre avis, ces techniques ne sont pas encore suffisamment mûres, même si, ici, les évolutions sont très rapides. Il nous semble plus accessible pour 
l'immédiat de donner ce rôle à l'animateur. Celui-ci, en effet, dans la plupart des méthodes de conception, ne joue aucun rôle technique. Il lui est donc possible de noter en permanence les différents critères et de les faire régulièrement valider par le groupe. En l'absence d'animateur officiel, c'est le secrétaire de la réunion ou le chef de projet qui devra tenir ce rôle. On peut prévoir explicitement un outil qui permette de capter les critères et d'obtenir un accord du groupe sur la captation de ces critères. L'idée ici est de produire deux résultats à la fin de la réunion: la solution et l'argumentation sur la solution avec les différentes conjectures qui s'y rapportent.

\section{Conclusion}

Cet article propose un cadre structuré pour capitaliser la logique de conception. Le mouvement conjecture-critère est la base de cette logique, dont cinq niveaux sont utilisés pour la représenter. Au terme de ce texte, nous proposons donc de mettre en œuvre des outils d'aide à la rationalisation de la conception. Ces outils seront constitués par une base de données portant sur des critères de conception qui sera reliée à la base de données produits. La mise en œuvre de ces bases de données dépend d'une instrumentalisation du processus tout à fait en accord avec la norme ISO 9001. Il nous semble, pour les raisons indiquées ci-dessus, que cette phase est nécessaire à une meilleure gestion du processus de conception. Une telle base de données est actuellement prototypée sur le logiciel CALC (Outil d'Aide à la Capitalisation de la Logique de Conception). Nous prévoyons de former des concepteurs et de tester sur des réunions de conception ces concepts et leur usage.

\section{Références}

Blanco, E., (1998), "L'émergence du produit dans la conception distribuée", Thèse de Doctorat, Université de Grenoble.

Duffy, A.H.B., Legler S., (1999), "Rationalising past designs for reuse", Proceedings of the $12^{\text {th }}$ International Conference on Engineering Design ICED99, (Editors: Lindemann, Birkhofer, Meerkamm, Vajna, ) vol.1, pp. 377-381, Munich, Germany.

Garro, O., Salaü, I., Martin P., (1995), "Distributed Design Theory and Methodology", Inf. ]. of Concurrent Engineering: research and applications, vol 3, number 1, pp. $43-54$.

Karsenty, L., (1994), "L'explication d'une solution dans les dialogues de conception", Thèse de l'Université Paris VII.

Karsenty, L., (2000), "Méthodes pour la création de mémoires de projet en conception", in Capitalisation des Connaissances en Conception, Actes de la Journée technique PRéCI, Sévenans, Mars 2000, pp. 1-12.

Laureillard P., Boujut J.F., Jeantet A. (1997), "Conception intégrée et entités de coopération", in Les objets de conception, Paris, ed. Hermes.

MacLean A., Young R., Bellotti V., Moran T., (1996), "Questions, Options, and Criteria: Elements of design space analysis", in Design rationale, LEA publishers, pp. 53-105. 
Mettavant, Y., Bousta A., Hickel, J., Pelliccia, S., (1999), "Informations sur la conception distribuée", Projet UTBM.

Ostrosi, E., (1998),"Contribution à l'étude d'une Méthode de Représentation et de Reconnaissance de 'Features' pour les systèmes avancés de CFAO mécanique", Thèse de Doctorat, Université Louis Pasteur de Strasbourg.

Tichkiewitch, S., (1991), "Integrated Computer Aided design for mechanical part", Advances in computer science application to machinery, Proceedings of the International Conference an CAD of Machinery, Pekin, China. 